\title{
An experimental study of a film cooling with swirling flow on the endwall of a high loaded 1st nozzle
}

\author{
K. Takeishi, Y. Oda \& S. Kondo \\ Department of Mechanical Engineering, Osaka University, Japan
}

\begin{abstract}
This paper describes an experimental study on film cooling effectiveness of circular and fan-shaped film cooling holes with a swirling film coolant injected through the endwall of a high-loaded 1st nozzle. The experiments have been conducted by using a two dimensional vane cascade, designed based on the firststage vane of $\mathrm{E}^{3}$ (Energy Efficient Engine) engine studied by a NASA project. The film cooling effectiveness on the endwall of the enlarged 1st nozzle of $E^{3}$ turbine was measured by using pressure sensitive paint (PSP) techniques. It appeared from the experimental results that the film cooling effectiveness of a circular hole was improved by increasing the angle $\theta$ of two impingement jets inside the cavity, which is used both to cool internal wall and to produce a swirling motion of film coolant. On the other hand, it was found that there exists an optimal jet angle between $\theta=10$ to 15 degrees in the case of a fan shaped film cooling hole. Thus the new film cooling method with swirling cooling air has been demonstrated to maintain its high film cooling effectiveness even under such a complicated flow field.
\end{abstract}

Keywords: gas turbine, heat transfer, film cooling, swirling flow, PSP.

\section{Introduction}

Gas turbines are used for aircraft propulsion and land-based power generation. Developments of turbine cooling technology play a critical role in increasing the thermal efficiency and power output of advanced gas turbines. Gas turbine's vanes and blades are cooled externally by film cooling. It is achieved by injecting relatively cooler air (film coolant) from the internal coolant passages out of the vane surface in order to form a protective layer between the blade 
surface and hot gas-path flow. The interaction between the film coolant and mainstream forms a shear layer, where the mixing of them occurs and leads to the decay of the film cooling performance along a vane surface.

It is important for film cooling to improve the cooling efficiency with an allowable coolant flow rate, because the excess use of the coolant can result in the decrease of thermal efficiency of gas turbines. Thus, it is required for new film cooling techniques to achieve wider and longer coverage of the coolant with a minimum coolant flow rate. When simplest circular holes are employed, it is known that the film coolant often penetrates into mainstream and generates a pair of kidney-shaped vortices by the interaction between mainstream and film cooling jet. The downwash of the vortices entrains the hot mainstream below the film coolant jet, and drastically decreases the film cooling effectiveness on turbine vane surfaces. Thus, fan-shaped film cooling has been one of the most attractive inventions attained by an incorporation of the exit shaping of the film holes, which results in lower momentum coolant injections with greater surface coverage.

There have been so many studies on film cooling. A review paper by Goldstein [1] summarized early works in this area and Bogard and Thole [2] reviewed latest one. Early investigations on film cooling were flows blowing through inclined discrete holes including Eriksen and Goldstein [3] and Bernsdorf et al. [4]. The efforts to improve film cooling efficiency by adopting film cooling holes with expanded exits have been made experimentally by Goldstein and Eckert [5], Bell et al. [6], Takeishi and Aoki. [7] and Yu et al. [8]. Excellent summary paper on shaped film cooling was published by Bunker [9].

Several new ideas to control anti-kidney vortex structure are presented. Kusterer et al. [10] investigated two film cooling arrangement with different compound angles, and Heidmann and Ekkad [11] proposed additional cooling air jets in a circular film cooling hole to control and to crash the kidney type vortex.

Takeishi et al. [12] have proposed a new film cooling method using a swirling coolant, and demonstrated it improves film cooling efficiency drastically by a wind tunnel test. In this study, we applied this method to the endwall of a high loaded turbine cascade, which is designed based on the first-stage vane of $\mathrm{E}^{3}$ (Energy Efficient Engine) engine studied by a NASA project, to examine the effect of the film cooling with swirling coolant in a realistic flow condition, where secondary flows and vortex systems exist as shown in Figure 1.

Figure 1 shows the conception of the three-dimensional flow field inside a first-stage turbine vane passage [13]. There is the so-called horseshoe vortex at the junction of the leading edge and the endwall. The pressure-side leg of the horseshoe vortex is combined with the low-momentum flow near the endwall to form what is known collectively as the passage vortex. The other leg is converted around the leading edge to the suction surface and remains close to the suction surface until it comes to the separation line of the endwall boundary layer. At the separation line, the suction-side leg of the horseshoe vortex lifts off the endwall and grows rapidly downstream along the suction surface adjacent to the passage vortex, as the so-called counter vortex. 


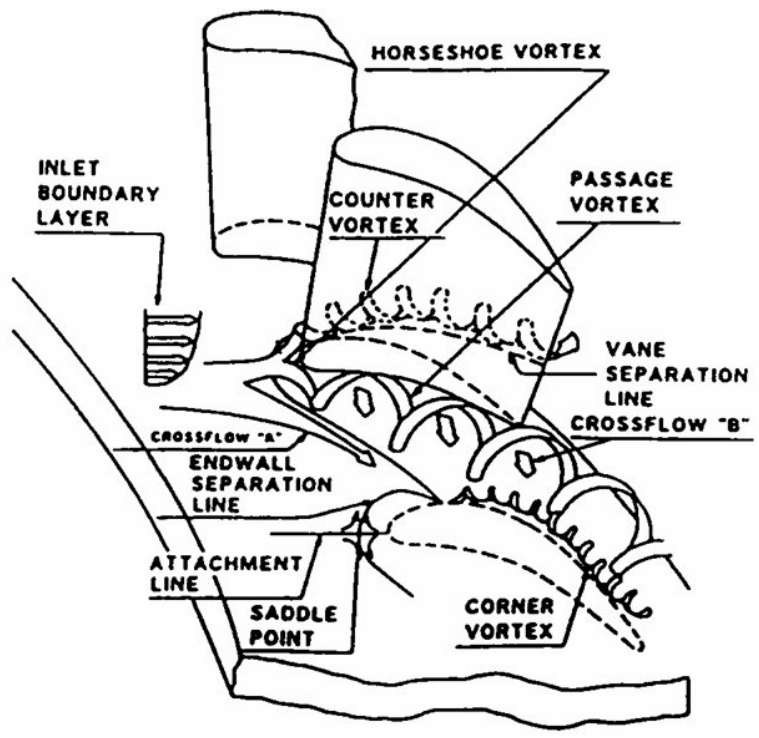

Figure 1: A secondary flow model near endwall.

\section{Experimental apparatus and measuring method}

\subsection{Low speed cascade test rig}

The experiment was conducted with a low-speed open-circuit, sector cascade wind tunnel. Figure 2 shows a schematic diagram of the test apparatus. The mainstream air is at room temperature with atmospheric conditions. The inlet velocity was measured by the Pitot tube mounted $100 \mathrm{~mm}$ upstream the leading edge of the vane. The inlet velocity was selected as $5 \mathrm{~m} / \mathrm{s}$ and the air is accelerated to $27.2 \mathrm{~m} / \mathrm{s}$ at the exit of the cascade under typical test conditions. The inlet velocity is selected as $5 \mathrm{~m} / \mathrm{s}$, which is accelerated to be $19.5 \mathrm{~m} / \mathrm{s}$ through the nozzle.

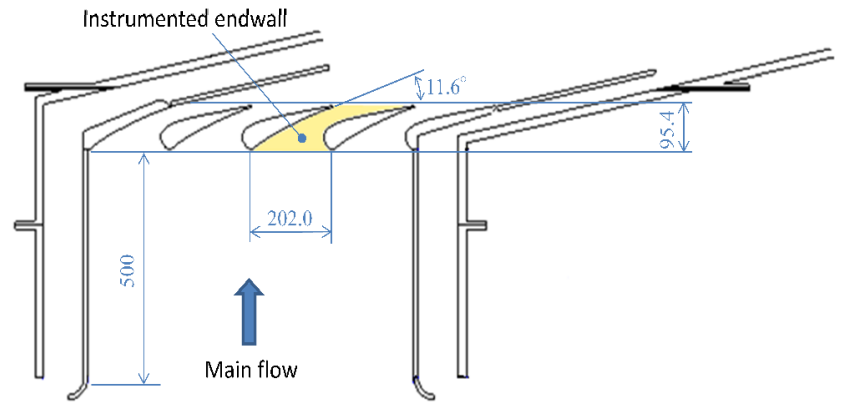

Figure 2: Geometry of a low-speed cascade test rig. 
The boundary layer thickness measured at a point $35 \mathrm{~mm}$ upstream the leading edge of the vane was $\delta_{.99}=7.1 \mathrm{~mm}$ on the testing endwall and the ratio to the vane height was $4.7 \%$. The turbulence intensity of the mainstream was $\tau=0.81 \%$ at the leading edge in the middle channel of the model vane. This value is low compared with that in actual engine conditions but the flow condition is acceptable when our aim is to investigate the effect of swirling coolant flow on film cooling effectiveness. The cascade was constructed with 5 vanes with an aspect ratio of $h / c=0.65$. The vane chord length and height are $230.2 \mathrm{~mm}$ and $150.0 \mathrm{~mm}$, respectively. The Reynolds number based on chord length and exit velocity is $4.1 \times 10^{5}$. The cascade geometry is given in Table 1 . The nozzles were made by ABS resin and the center nozzle was instrumented static pressure taps to measure aerodynamic conditions.

Table 1: $\quad$ Geometry and dimensions of cascade.

\begin{tabular}{|c|c|}
\hline Scale & 2.19 \\
\hline Axial Chord, $C_{a x}[\mathrm{~mm}]$ & 95.4 \\
\hline Chord, $C[\mathrm{~mm}]$ & 224.6 \\
\hline Span, $S[\mathrm{~mm}]$ & 150.0 \\
\hline Pitch, P $[\mathrm{mm}]$ & 202.0 \\
\hline L.E. \& T. E. Diameter $[\mathrm{mm}]$ & $29.2 \& 3.6$ \\
\hline${\text { Inlet } \& \text { Exit Angle }\left[{ }^{\circ}\right]}$ & $90.0 \& 11.6$ \\
\hline
\end{tabular}

The configuration and dimensions of the film cooling holes are shown in Figure 3. Swirling coolant flow is made in a cavity that is located in a plenum before entering into a film cooling hole. A schematic view of the cooling structure is shown in Figure 4. The cross-section shape of the cavity is hexagonal, because it can be set successively without void space in real applications. It has two impingement jet holes, and they are inclined at degree toward the vertical direction. Two impingement jets generate swirling flows inside the cavity, and this swirling flow enters into a circular film cooling hole keeping the angular momentum. This cooling structure was mounted at the endwall of the above mentioned low speed cascade. The flow channel for measurements was selected between the pressure side of the centre vane and the

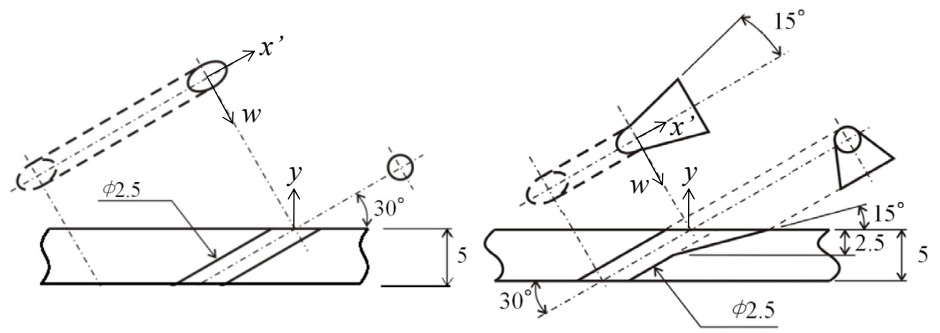

Figure 3: Geometry of film cooling holes. 


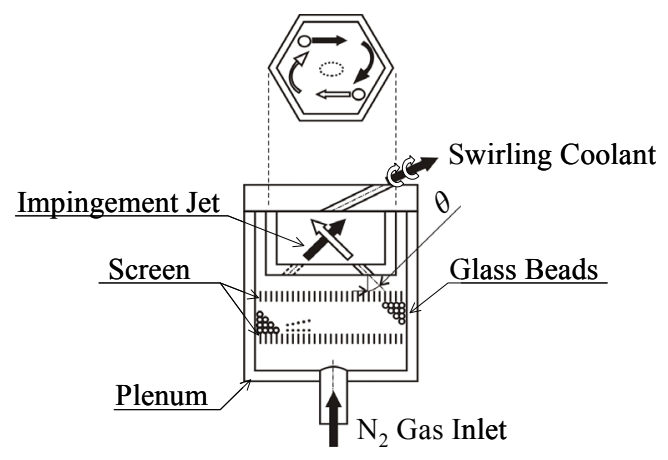

Figure 4: Structure of film cooling with swirling flow.

suction side of the next vane as shown in Figure 2. There is a pressure difference between the pressure and suction surfaces, and a secondary flow is generated by this pressure differences. The flow direction of this secondary flow is not the same as main flow. The film cooling model can turn on the centre of the film cooling hole and we define a compound angel as $\alpha$, which is the angle between the secondary flow stream line and the direction of film coolant injection. The test model of film cooling hole was mounted on the endwall of the low speed cascade where the main stream velocity is about $20 \mathrm{~m} / \mathrm{s}$, in order to match the local flow condition over the cooling hole with the film cooling test by using a flat plate in a low speed wind tunnel [12]. Figure 5 shows the location of the film cooling hole at the endwall of the low speed cascade rig. The left side of Figure 5 shows the streamline on the endwall, which was calculated by Large Eddy Simulation (LES). Based on this numerical data, the location and direction of the film cooling hole was determined as shown in the right side of Figure 5. The area encircled by a dotted line in Figure 5 shows the measurement area of film cooling effectiveness shown in the figures in results and discussion.
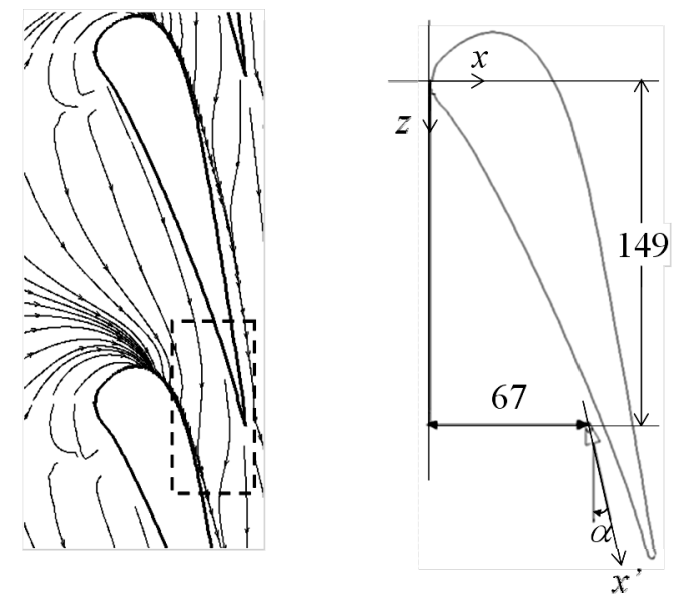

Figure 5: Streamline on the endwall and cooling hole location and direction. 
In the present study, the angle of two impingement jets inside the hexagonal cavity was changed as $\theta=0^{\circ}, 5^{\circ}, 10^{\circ}, 15^{\circ}, 20^{\circ}, 30^{\circ}, 40^{\circ}$ at $\alpha=0^{\circ}$. The experimental conditions are shown in Table 2.

Table 2: $\quad$ Experimental conditions of film cooling tests.

\begin{tabular}{|c|c|}
\hline Mainstream velocity, $u_{\infty}[\mathrm{m} / \mathrm{s}]$ & 20 \\
\hline Turbulence intensity, $\tau[\%]$ & 0.36 \\
\hline Boundary layer thickness, $\delta[\mathrm{mm}]$ & 25 \\
\hline Blowing ratio, $M[-]$ & $0.5,1.0,1.5,2.0$ \\
\hline Film cooling hole diameter, $d[\mathrm{~mm}]$ & 5 \\
\hline Impingement jet angle, $\theta\left[^{\circ}\right]$ & $0,5,10,15,20,30,40$ \\
\hline
\end{tabular}

\subsection{PSP methods}

In this study, PSP was used to measure the film cooling effectiveness distribution on the wall. PSP is an optical pressure sensor. It uses a special pigment that changes its luminescence intensity by the reaction with oxygen molecules. The change of luminescence intensity is caused by optical quenching of the pigment by oxygen molecules. Therefore, we can measure a change of the concentration of oxygen by the luminescence intensity of the pigment. By using air (concentration of oxygen is $21 \%$ ) for mainstream and nitrogen (concentration of oxygen is $0 \%$ ) for film cooling jet, the concentration distribution of oxygen, which is determined by the mixing of mainstream (air) and coolant (nitrogen), can be measured on the wall downstream of the film cooling hole. The film cooling effectiveness was defined by the following equation:

$$
\eta=\frac{T_{\infty}-T_{a w}}{T_{\infty}-T_{c}}
$$

where $T_{\infty}$ and $T_{c}$ are temperatures of mainstream and coolant in the plenum respectively; $T_{a w}$ is the adiabatic wall temperature.

Then, using an analogy between heat/mass transfer, Eq. (1) is changed to Eq. (2).

$$
\eta=\frac{C_{\infty}-C_{w}}{C_{\infty}-C_{c}}
$$

where $C_{\infty}$ and $C_{c}$ are concentration of the oxygen in mainstream and plenum chamber respectively; $C_{w}$ is the concentration of the oxygen in the film cooling air just above the wall.

The blowing ratio, which is defined as Eq. (3) was controlled by changing the film cooling air flow rate.

$$
M=\frac{\rho_{c} u_{c}}{\rho_{\infty} u_{\infty}}
$$




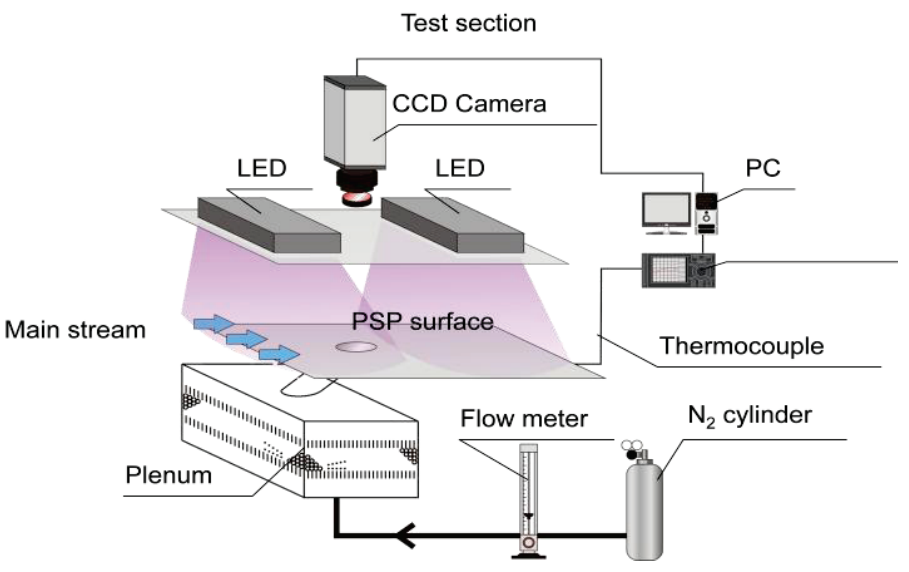

Figure 6: Measuring system of film cooling effectiveness by PSP technique.

The wall of the wind tunnel is painted with PSP (ISSI PtTfPP FIB-UF405). Nitrogen gas for film cooling is supplied from nitrogen gas cylinder. Thus, $C_{c}$ and $C_{\infty}$ are $0 \%$ and $21 \%$, respectively, and $C_{w}$ is measured by PSP using the following experimental setup.

Figure 5 shows the schematic layout of PSP to measure the film cooling effectiveness. LED light sources with wave length of $405 \mathrm{~nm}$ were used as an excitation light source. The images were taken with a CCD camera (Hamamatsu photonics C9440-05C $1344 \times 1024$ pixels, 12bit) located at the opposite side of PSP painted wall. Here, the light except phosphorescence with wave length of $650 \mathrm{~nm}$ was cut by a band pass filter. The images were digitally stored on hard disks using the acquisition software HIPIC 8.30. The three images, a background image, an image when wind tunnel was pausing and an image when wind tunnel was operating, were captured. The set of 20 images were captured in each case, and they were averaged. PSP's luminescence intensity is affected by its temperature and hence the temperature of the wall during the experimentation was monitored by K-type thermocouples. In the plenum, there were screen and glass beads to prevent direct entrance of the supplied air into the impingement cooling holes.

An uncertainty analysis was performed on the film cooling effectiveness measured by PSP. The PSP method is sensitive to temperature. In this experiment, the maximum error of temperature measurement is within $\pm 0.1^{\circ} \mathrm{C}$, and it affects the uncertainty of film cooling effectiveness as $\pm 0.7 \%$. Consequently, the total experimental uncertainty sums up to $1.6 \%$ in the area where the film cooling effectiveness $\eta$ is larger than 0.4 , but it becomes $7.2 \%$ in the area $\eta \cong 0.1$ because the light of phosphorescence becomes weak in the area where the film cooling effectiveness is less than 0.1 . 


\section{Results and discussion}

Figures 7 and 8 show the distribution of film cooling effectiveness of the circular hole in the cascade rig without swirling from $M=0.5$ to 2.0 in Figure 7 and with swirling from $\theta=10^{\circ}$ to $40^{\circ}$ at $M=1.5$ in Figure 8. It appears from these figures that film cooling effectiveness distribution downstream is different in each case. Figure 7 shows that the film cooling effectiveness decreases due to the penetration of the film cooling air to the mainstream, as the blowing ratio $M$ increases. In case (a) for $\theta=0^{\circ}$ and $M=0.5$, film cooling effectiveness distribution downstream tends to be drifted to the suction surface of the vane by the secondary flow from the pressure side to suction side. On the other hand, in case (d) with $M=2.0$, film cooling effectiveness distribution downstream tends to be less drifted to the suction side compared to case (a) due to the higher

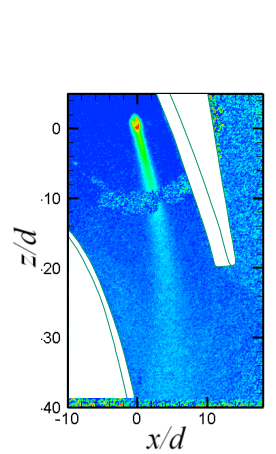

(a) $M=0.5$

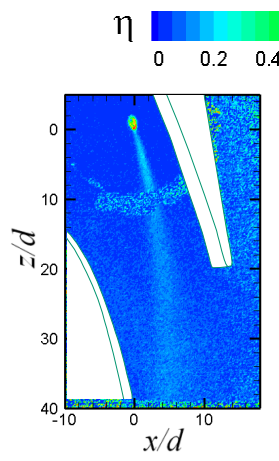

(b) $M=1.0$

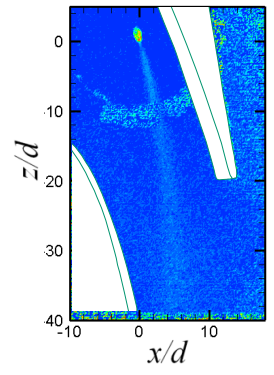

(c) $M=1.5$

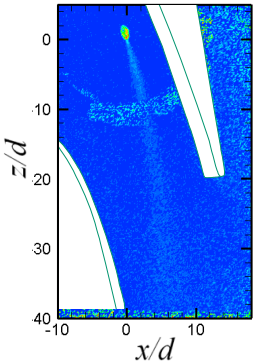

(d) $M=2.0$

Figure 7: Film cooling effectiveness contours of a circular hole with $\theta=0^{\circ}$ on the endwall.
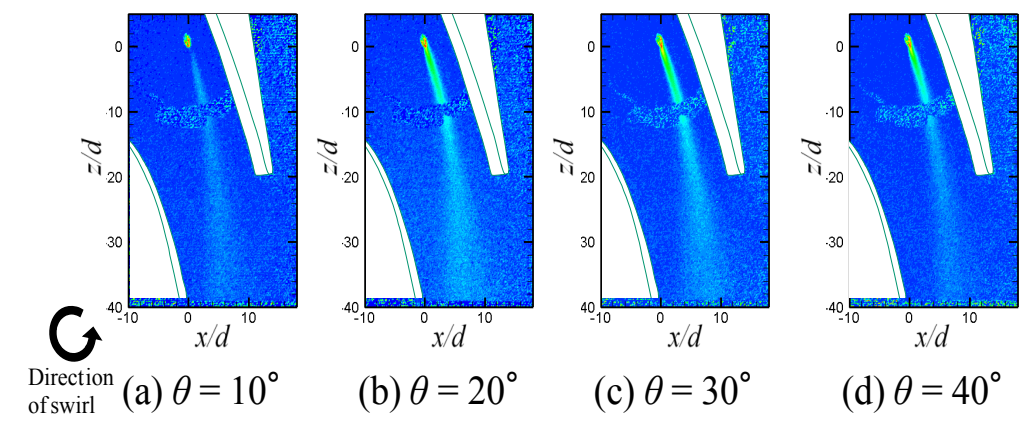

(b) $\theta=20^{\circ}$

(c) $\theta=30^{\circ}$

(d) $\theta=40^{\circ}$

Figure 8: Film cooling effectiveness contours of a circular hole at $M=1.5$ on the endwall. 
blowing ratio $M$. Figure 8 shows that the film cooling effectiveness attains maximum at $\theta=20^{\circ}$ and this optimal angle is shifted to larger side from that of flat plate with $M=1.5$.

Figures 9 and 10 show the distribution of film cooling effectiveness of the fan-shaped hole in the cascade rig without swirling from $M=0.5$ to 2.0 in Figure 9 and with swirling from $\theta=5^{\circ}$ to $20^{\circ}$ at $M=1.5$ in Figure 10 . Since the jet penetration is suppressed in the fan-shaped hole, film cooling effectiveness is improved with the increase of the blowing ratio from $M=0.5$ to 2.0 in the noswirl case as shown in Figure 9. Figures 10 (b) and (c) show a basically similar distribution, but spanwise spreading of the coolant is enhanced downstream by the swirling motion in the case of $\theta=15^{\circ}$. When the swirl is further increased at $\theta=20^{\circ}$, the core region of coolant jet is shifted toward pressure side by the strong swirl inside the hole. This swirling flow still remains in the coolant out of the hole, and enhance the spanwise spreading of the coolant downstream.
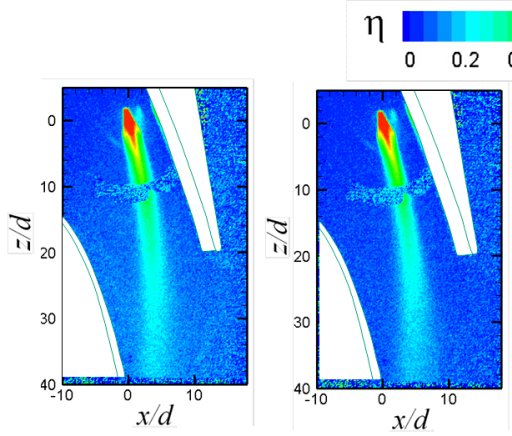

(a) $M=0.5$

(b) $M=1.0$
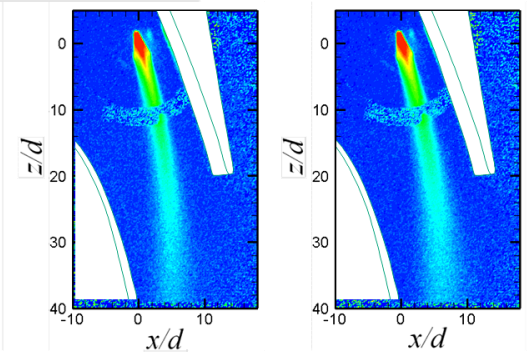

(c) $M=1.5$

(d) $M=2.0$

Figure 9: Film cooling effectiveness contours of a fan-shaped hole with $\theta=0^{\circ}$ on the endwall.

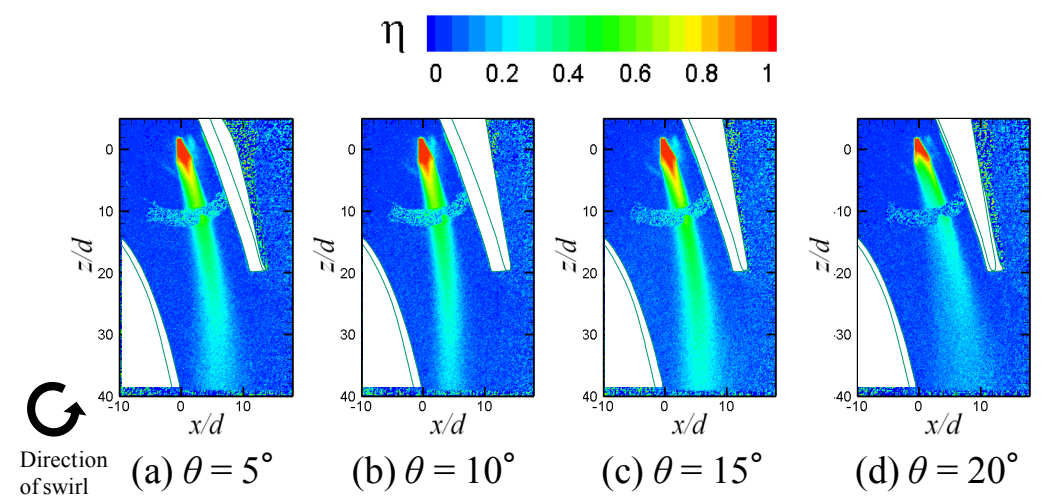

Figure 10: Film cooling effectiveness contours of a fan-shaped hole at $M=1.5$ on the endwall. 
Figure 11 shows the averaged film cooling effectiveness $\bar{\eta}$ for the cases of circular hole and fan-shaped hole at $x^{\prime} / d=5$ respectively. It appears from these figures that the film cooling effectiveness attains maximum at $\theta=20^{\circ}$ in case of circular hole at $M=0.5-1.5$ and this optimal angle is shifted to larger angle of $\theta=30^{\circ}$ at $M=2.0$. In case of fan-shaped hole, the film cooling effectiveness attains maximum at $\theta=15^{\circ}$. In both cases, the averaged film cooling effectiveness tends to increase with adding swirling motion to the film cooling air, and attains its maximum effectiveness near $\theta=15^{\circ}$ to $20^{\circ}$ and decrease the effectiveness with an increase of swirling motion due to the hot mainstream gas entrained under the film cooling air by the swirling motion.

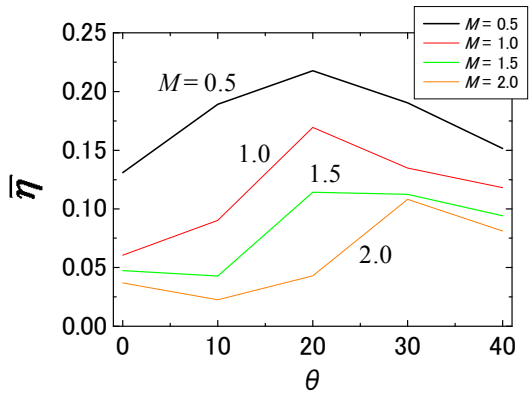

(a) Circular hole

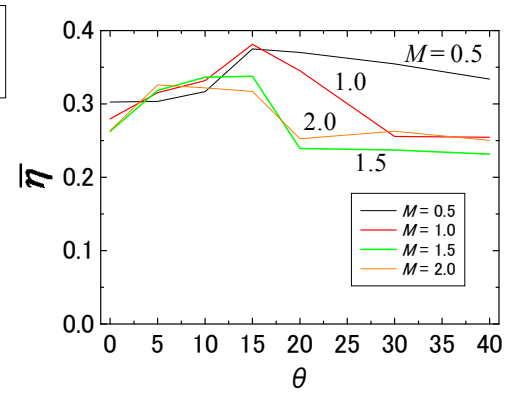

(b) Fan-shaped hole

Figure 11: Spanwise averaged film cooling effectiveness at $x^{\prime} / d=5$ on the endwall.
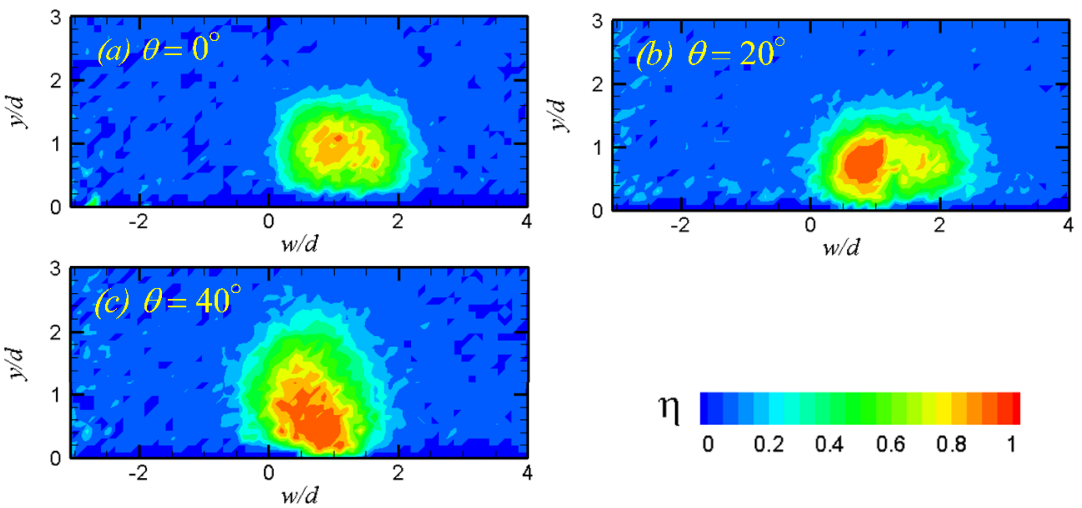

Figure 12: Non-dimensional concentration contours of a circular hole with $M$ $=1.5$ and $a=0^{\circ}$ at $x^{\prime} / d=5$ on the endwall.

Figures 12 and 13 show the time-averaged non-dimensional concentration distribution of acetone at the cross section at $x^{\prime} / d=5$ obtained by acetone LIF [12]. It appeared from Figure 12 that the penetration of film cooling air jet into the mainstream occurred at high blowing ratio of $M=1.5$ with non-swirling 
coolant flow case. Increasing the swirling motion of the coolant flow up to $\theta=20^{\circ}$, the half part of the coolant impinges and adheres on the endwall and attains higher film cooling effectiveness. But when the swirling motion of the coolant flow is increased up to $\theta=40^{\circ}$ as shown in Figure 12 (c), the swirling motion is so strong to roll up the hot mainstream between the film cooling air and the endwall. As the result, the film cooling effectiveness of a circular hole at $M=1.5$ with $\theta=40^{\circ}$ attains poor film cooling effectiveness as already shown in Figure 11 (a).
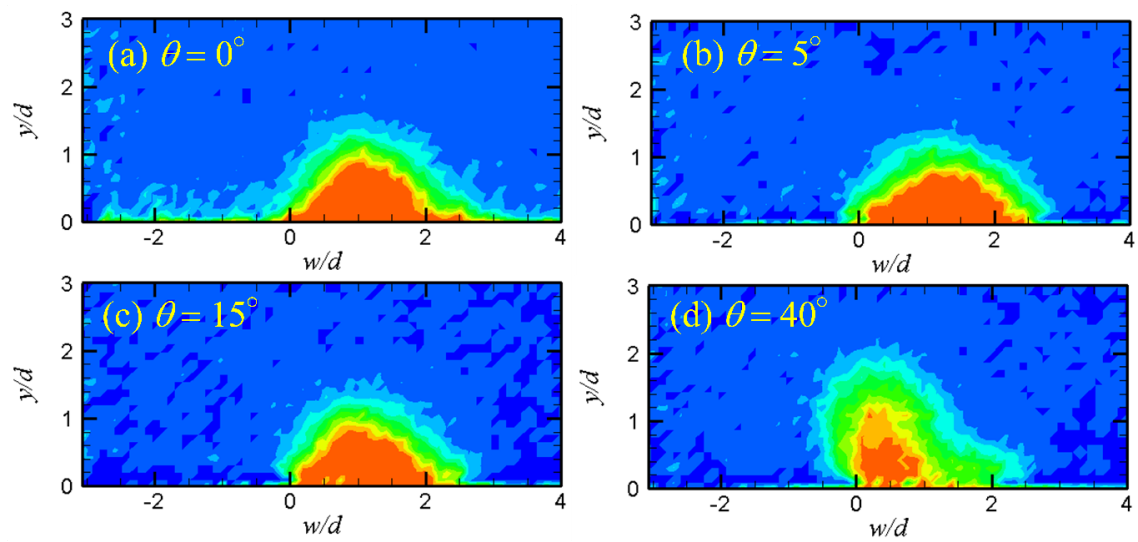

Figure 13: Non-dimensional concentration contours of a fan-shaped hole with $M=1.5 a=0^{\circ}$ at $x^{\prime} / d=5$ on the endwall.

The fan-shaped film cooling attains higher film cooling effectiveness as it appeared from Figure 13(a) even at high blowing ratio $M=1.5$. When the swirling motion of the coolant flow is increased up to $\theta=15^{\circ}$ as shown in Figure 13(b) and (c), the film cooling air adheres tightly on the endwall and increase the film cooling effectiveness little by little. But strong swirling motion at $\theta=40^{\circ}$ rolls up the main hot gas under the film cooling air and decreases the film cooling effectiveness as it is clearly shown in Figure 13(d).

In case of fan-shaped film cooling, film cooling layer generated by the swirling coolant interacts with secondary flow and becomes unstable at far downstream at $x^{\prime} / d=20$ and the averaged film cooling effectiveness shows zigzag shape as like shown in Figure 11(b).

\section{Conclusions}

The new invention to improve the film cooling effectiveness by using swirling coolant is applied to the endwall of a low speed cascade. The following conclusions were obtained through these experimental research works.

1. Film cooling of a circular hole with swirling coolant improved the film cooling effectiveness on the endwall of low speed cascade, where complicated vortex system and secondary flow exist. 
2. The film cooling effective of a fan-shaped film cooling hole attains maximum value at $\theta=15^{\circ}$ at high blowing ratio of $M=1.5$. It is estimated that there is an optimal condition depending on parameters, such as film cooling geometry, blowing ratio, yaw angle to secondary flow, strength of swirl, etc.

3 . The swirling motion of film coolant is effective to improve the film cooling effectiveness in a wide range of $\theta$ for a circular hole, and in a narrow range of $\theta$ for a fan-shaped hole.

\section{References}

[1] Goldstein, R. J., 1971, "Film Cooling", Advances in Heat Transfer, Vol. 7, pp. 321-379.

[2] Bogard, D. G. and Thole, K. A., 2006, "Gas Turbine Film Cooling", J. of Propulsion and Power, 22, pp. 249-270.

[3] Eriksen, V, L. and Goldstein, R. J., 1974, "Heat Transfer and Film Cooling Following Injection Through Inclined Circular Tubes”, J. of Heat Transfer, pp. 239-245.

[4] Bernsdorf, S., Rose, M. G. and Abhari, R. S., 2005, "Modeling of Film Cooling - Part I: Experimental Study of Flow Structure", ASME Paper No. GT2005-68783.

[5] Goldstein, R. J. and Eckert, E. R. G., 1994, "Effect of hole geometry and density on three-dimensional film cooling", Int. J. Heat and Mass Transfer, 17, pp. 595-607.

[6] Bell, C. M., Hamakawa, H. and Ligrani, P. M., 2000, "Film Cooling From Shaped Holes", J. of Turbomachinery, 122, pp. 224-232.

[7] Takeishi, K., and Aoki, S., 2001, "Contribution of Heat Transfer to Turbine Blades and Vanes for High Temperature Industrial Gas Turbines: Part 1, Film Cooling”, Ann. N. Y. Acad. Sci., Vol. 934, pp. 305-312.

[8] Yu, Y, Yen, C. -H. Shin, T. I. -P. and Chyu, M. K. 2000, "Film Cooling Effectiveness and Heat Transfer Coefficient Distribution Around Diffusion Shaped Holes", J. of Heat Transfer, 124, pp. 820-827.

[9] Bunker, R. S., 2005, "A Review of Shaped Hole Turbine Film-Cooling Technology", J. of Heat Transfer, 127, pp. 441-453.

[10] Kusterer, K., et al. 2009, "A parametric study on the influence of the lateral ejection angle of double-jet holes on the film cooling effectiveness for high blowing ratios", ASME Paper GT2009-59321.

[11] Heidmann, J. D. and Ekkad, S., 2007, "A novel anti-vortex turbine filmcooling hole concept", ASME paper, GT2007-27528.

[12] Takeishi, K., Oda, Y., Egawa, Y. and Kitamura T., 2010, "Film Cooling with Swirling Coolant Flow," WIT Transactions on Engineering Sciences, 68, pp. 189-200.

[13] Takeishi, K., Matsuura, M., Aoki, S., and Sato, T., 1990, “An Experimental Study of Heat Transfer and Film Cooling on Low Aspect Ratio Turbine Nozzles, Trans. of ASME, J. of Turbomachinery, 112, pp. 488-496. 